spontaneous abortion: comparing expectant management with surgical evacuation. Human Reproduction 1996; 11: 1767-70.

6. Smith LF. Women's experiences of three early miscarriage management options - a qualitative study. British Journal of General Practice 2006; 56: 198-205.

7. Wiebe E, Janssen P. Conservative management of spontaneous abortions. Women's experiences. Canadian Family Physician 1999; 45: 2355-60.

8. Wieringa-de W M, Ankum WM, Bonsel GJ, Vos J, Biewenga $\mathrm{P}$, Bindels PJE. The natural cause of spontaneous miscarriage: analysis of signs and symptoms in 188 expectantly managed women. British Journal of General Practice 2003; 53: 704-8

9. Swanson KM, Chen HT, Graham C, Wojnar DM, Petras A. Resolution of depression and grief during the first year after miscarriage: a randomised controlled clinical trial of couplesfocused interventions. Journal of Women's Health 2009; 18: 1245-57.

10. Smith LFP, Ewings PD, Quinlan C. Incidence of pregnancy after expectant, medical, or surgical management of spontaneous first trimester miscarriage: long term follow up of miscarriage treatment (MIST) randomised controlled trial. British Medical Journal 2009; 339: b3827.

11. Waard M, Hartman EE, Ankum WM, Reitsma JB, Bindels PJ, Bonsel GJ. Expectant management versus surgical evacuation in first trimester miscarriage: health-related quality of life in randomised and non-randomised patients. Human Reproduction 2002; 17: 1638-42

12. Stirtzinger R, Robinson GE. The psychologic effects of spontaneous abortion. Canadian Medical Association Journal 1989; 140: 799-805

\title{
Correlation of clinical, parasitological and histopathological diagnosis of cutaneous leishmaniasis in an endemic region in Sri Lanka
}

\author{
R R Ranawaka ${ }^{1}$, P H Abeygunasekara ${ }^{2}$, H S Weerakoon ${ }^{1}$ \\ (Index words: L. donovani, cutaneous leishmaniasis, clinical diagnosis, parasitology, Sri Lanka)
}

\begin{abstract}
Introduction Diagnosis of cutaneous leishmaniasis (CL) largely depends on the clinical appearance of the lesions in endemic areas.

Objectives The aim of this study was to correlate the clinical diagnosis with positive laboratory tests used for the identification of leishmania parasite (stained smears and histopathology) and therapeutic response.

Methods 114 clinically suspected patients (190 lesions) were studied. They were diagnosed as typical clinical cases $(87.4 \%)$ and clinically suggestive cases $(12.6 \%)$ on the basis of criteria for clinical diagnosis. Slit-skinsmear and histopathology were performed in all patients.

Results Out of 103 who were clinically diagnosed, 62 $(60.2 \%)$ were confirmed parasitologically. Out of 41 who were negative for both smear and histology, 34 (33\%) had supportive histology and the diagnosis was supported by good treatment response in 37 (35.9\%). Hence, the clinical diagnosis was $93.2 \%$ accurate and this increased to $96 \%$ after observing the good response to therapy.
\end{abstract}

Conclusions Considering the magnitude of the problem, limited resources, and clinical accuracy of $96 \%$, clinical diagnosis by a dermatologist appears to be reliable enough in diagnosing $\mathrm{CL}$ lesions in endemic areas in Sri Lanka.

Ceylon Medical Journal 2012; 57: 149-152

\section{Introduction}

Cutaneous leishmaniasis (CL) is a growing public health problem in several parts of the world, including Sri Lanka [1-4]. The causative organism is identified as Leishmania donovani zymodeme MON-37 [5]. Although occasional cases are reported from all the provinces, Southern and North-Central provinces are endemic reporting more than 1000 cases annually $[2,3]$. The diagnosis of CL largely depends on the clinical appearance, especially in endemic areas. As a general rule in endemic areas of the world any boil that is present for several weeks and does not respond to ordinary therapy should be considered as CL unless proven otherwise [6]. A diagnostic challenge arises when the lesions appear in

Departments of ${ }^{1}$ Dermatology, ${ }^{2}$ Pathology, Teaching Hospital, Anuradhapura, Sri Lanka.

Correspondence: RRR, e-mail: <ranthilaka37@yahoo.com>. Received 23 May and revised version accepted 23 August 2012. Competing interests: none declared. 
nonendemic areas, when the clinical picture is distorted, or when an atypical variant is seen.

Giemsa stained smears obtained from the lesions are a rapid means of diagnosis. A smear can be made in different ways; a slit smear, the impression or touch method, scalpel scraping, or by fine-needle aspiration [6, 7]. The histological picture in CL differs according to the stage of infection and the clinical type. It is important to search for amastigotes, which are diagnostic [6-8]. Intralesional sodium stibogluconate, intralesional hypertonic sodium chloride solution and liquid nitrogen cryotherapy are effective on cutaneous leishmaniasis $[9,10]$.

The aim of this study was to correlate the clinical diagnosis with positive laboratory tests used for the identification of leishmania parasite (stained smears and histopathology) and therapeutic response.

\section{Methods}

The study was conducted at Dermatology Unit, Teaching Hospital, Anuradhapura over 10 months from January to September 2009. 114 patients (190 skin lesions) who were clinically suspected of having CL were evaluated. The clinical diagnosis was made by the dermatologist and the lesions were grouped into typical clinical cases and clinically suggestive cases. Typical clinical cases were those who had discrete papules, nodules or non-healing noduloulcerative skin lesions if they met at least four of the following five clinical criteria:

1. origin from a known endemic area, or have visited an endemic area during the period of onset of the lesion

2. painless and non itchy lesions

3. duration of several weeks to a month

4. resistant to conventional antibiotics

5. history of similar lesions in the household or locality

Atypical CL lesions, i.e. non healing ulcers, punchedout ulcers and large plaques having four of the five above clinical criteria were considered as clinically suggestive lesions. Clinical accuracy was analysed on the basis of percentage of clinically diagnosed cases that proved to be true cases of CL by laboratory investigations and/or by good therapeutic response.

Leishmania slit-skin smear and histopathology were performed on all patients. When multiple lesions were present, these tests were performed from one selected lesion. PCR and culture were not performed routinely. A3 $\mathrm{mm}$ slit at the lesion edge was made with a sterile scalpel while the lesion was strongly held between fingertips to form a bloodless field. The smears were placed on glass slides, fixed in methanol, and stained with Giemsa stain, and microscopically examined for the presence of amastigotes (Leishman-Donovan (LD) bodies).

Skin biopsy from the peripheral part of the skin lesion was obtained. These were routinely processed; 4-5 micron thick sections were stained with haematoxylin-eosin stain and examined under light microscopy. Histopathological specimens which demonstrated LD bodies were considered as histology positive cases. Skin biopsies which showed one of the recognisable histological patterns seen in CL without LD bodies were grouped as supportive histology [8].

Patients were treated either with intralesional sodium stibogluconate $(\mathrm{n}=6)$, hypertonic sodium chloride solution $(n=70)$ or liquid nitrogen cryotherapy $(n=27)$.

\section{Results}

The clinico-epidemiological profile of the study population is given in Table 1. Majority were men (M:F = 2.5:1). The age ranged from $4-80$ years (median 35.4 years). $79.4 \%$ were local residents who had acquired the disease from the vicinity. The duration of the lesions varied from 4 weeks to 5 years (median 9.2 months). Majority (70\%; $n=74)$ had a single lesion while only $6.6 \%(n=7)$ had more than 4 lesions (maximum up to 8 lesions). The most common site of the lesions was the face (27\%), followed by forearm (21\%), leg (20\%), trunk (17\%) and arm (15\%). Most frequent clinical presentation was the nodulo-ulcerative lesions (50\%), followed by papules $\leq 1 \mathrm{~cm}$ diameter $(29.7 \%)$.

The results of parasitological investigations are given in Table 2. Out of 114 patients who were included in our study, 103 were clinically diagnosed as cutaneous leishmaniasis by the dermatologist. Out of $103,87.4 \%$ $(n=90)$ were typical clinical cases while $12.6 \%(n=13)$ were clinically suggestive cases. There were $14.6 \%(n=15)$ patients who were slit-skin-smear (SSS) positive but histopathology was negative for LD bodies; $26.2 \%(n=27)$ were histopathology positive but SSS negative; $19.4 \%$ $(n=20)$ were both SSS and histopathology positive. Therefore total parasite positivity in the study group was $60.2 \%(\mathrm{n}=62)$. Overall, slit-skin-smear (SSS) positivity was $33.9 \%(35 / 103)$, while histopathology showing parasite positivity was $45.6 \%(47 / 103)$.

Out of 41 cases in whom both SSS and histopathology were negative, $33 \%(n=34)$ had supportive histology. Therefore clinical accuracy before therapy was $93.2 \%(96 / 103)$. Following standard therapy 37 of 41 got cured and clinical accuracy increased to $96 \%$ after observing the good response to therapy.

Following three histological patterns were seen in this study group.

(a) dense granulomatous infiltrate comprising epithelioid cells, lymphocytes, plasma cells, and histiocytes with numerous LD bodies ( $\mathrm{n}=30: 29.1 \%$ );

(b) dense inflammatory infiltrate comprising epithelioid cells, lymphocytes, plasma cells with occasional histiocytes containing a few LD bodies ( $n=17: 16.5 \%)$;

(c) dense inflammatory infiltrate comprising epithelioid cells, histiocytes, lymphocytes/plasma cells without LD bodies $(\mathrm{n}=34: 33 \%)$.

The two groups (a) and (b) which showed LD bodies were considered as histopathologically positive cases, while (c) which had granulomatous inflammation without LD bodies was considered as supportive histology. 
Table 1. Clinico-epidemiological profile of the study group

*Total may differ due to missing data

\begin{tabular}{|c|c|}
\hline & $\begin{array}{c}\text { Number } \\
\text { (percentage) }\end{array}$ \\
\hline \multicolumn{2}{|l|}{ Occupational distribution } \\
\hline Farmers & $28(25)$ \\
\hline Military personnel & $23(20.5)$ \\
\hline School children & $22(19.3)$ \\
\hline Housewives & $9(7.9)$ \\
\hline Home guards & $7(5.9)$ \\
\hline Teachers & $3(2.4)$ \\
\hline Others & $21(18.5)$ \\
\hline \multicolumn{2}{|l|}{ Clinical presentation } \\
\hline papules ( $\leq 1 \mathrm{~cm}$ diameter $)$ & $39(29.7)$ \\
\hline nodules $(1-2.5 \mathrm{~cm}$ diameter $)$ & $9(6.8)$ \\
\hline nodulo-ulcerative lesion & $66(50)$ \\
\hline plaques $(>2.5 \mathrm{~cm}$ diameter $)$ & $9(6.8)$ \\
\hline $\begin{array}{l}\text { chronic non-healing ulcers with undermined } \\
\text { edges without surrounding induration }\end{array}$ & $3(2.3)$ \\
\hline \multicolumn{2}{|l|}{ Site of the lesions } \\
\hline Face & $34(27)$ \\
\hline Arm & $19(15)$ \\
\hline Forearm & $27(21)$ \\
\hline Lower limb & $25(20)$ \\
\hline Trunk & $22(17)$ \\
\hline lesions on multiple sites & 6 \\
\hline
\end{tabular}

Table 2. Histopathological grade and smear positive status

\begin{tabular}{lcc}
\hline $\begin{array}{l}\text { Histopathological } \\
\text { grade }\end{array}$ & $\begin{array}{c}\text { Smear } \\
\text { positive status }\end{array}$ & $\begin{array}{c}\text { Smear } \\
\text { negative status }\end{array}$ \\
\hline $\begin{array}{l}\text { LD bodies positive in } \\
\text { skin biopsy ( } \mathrm{n}=47)\end{array}$ & $19.4 \%(\mathrm{n}=20)$ & $26.2 \%(\mathrm{n}=27)$ \\
$\begin{array}{l}\text { LD bodies negative in } \\
\text { skin biopsy }\end{array}$ & $14.6 \%(\mathrm{n}=15)$ & $39.8 \%(\mathrm{n}=41)$ \\
Total (n=103) & $33.9 \%(\mathrm{n}=35)$ & $66 \%(\mathrm{n}=68)$ \\
\hline
\end{tabular}

\section{Discussion}

Cutaneous leishmaniasis had been an endemic disease in Anuradhapura district in Sri Lanka for nearly 15 years [3]. But until 2007 skin smears were performed either at Department of Parasitology, Faculty of Medicine Colombo (224 km) or Faculty of Medicine Peradeniya (110 $\mathrm{km}$ ). The cost for this was nearly SL Rupees 2000 (approx US\$20) per patient, and the patients had to spend three working days for this investigation.
The clinical manifestations of leishmaniasis are not always pathognomonic and could mimic several other conditions. For example insect bites, tropical ulcers, leprosy, lupus vulgaris, cutaneous sarcoidosis, subcutaneous mycoses and skin cancers could mimic CL. Diagnosing CL may be challenging when the lesions appear in a non-endemic area, when superadded infection or home-made remedies distort the clinical picture, or when an atypical lesion is seen. In such cases laboratory diagnostic techniques such as skin smears, culture for parasites, histopathology and PCR facilitate the diagnosis.

All these traditional laboratory methods (stained smears, culture, and histopathology) had limitations, including low sensitivity, the requirement for culture facilities, and their time-consuming nature [6]. Demonstration of parasites in skin smears, skin biopsy and culture were confirmatory. Modern methods for the diagnosis of CL, such as immunofluorescence, immunohistochemistry, use of monoclonal antibodies, DNA probes, polymerase chain reaction and electronmicroscopic studies were sensitive methods, but were expensive [10-13]. PCR technique is highly specific (100\%) and sensitive (92.5\%) for the diagnosis of CL [11].

Slit-skin-smear positivity in this study group was low (33.9\%) compared to published data. When performed by experienced hands, this simple and cheap procedure can be employed as a rapid and economical means of diagnosis with accuracy ranging from $53.3 \%$ to $84.2 \%$ [6, 7]. Slide touch skin biopsy method show better results $(84.2 \%$ positivity) than slit-skin smear method (66.7\% positivity) [7]. Histological patterns seen were similar to those described with CL caused by L. major and L. tropica $[6,7]$. Histopathologic examination had shown positivity ranging from $59.6 \%$ to $68.5 \%$ in published data [7]. Histopathology showing $45.6 \%$ parasite positivity and further $33 \%$ giving supportive histology (overall 78.6\%) was encouraging in our study.

According to this study, clinical diagnosis by a dermatologist, supported by simple, cheap, and easy tests, such as smears performed locally reaches an accuracy of $96 \%$ and is reliable enough in diagnosing typical CL lesions in endemic areas in Sri Lanka. Histopathology should be reserved for doubtful lesions and atypical CL lesions.

\section{Acknowledgements}

We thank Professor J. Sarath Edirisinghe, Faculty of Medicine, Rajarata University for training Medical Laboratory Technicians of Teaching Hospital Anuradhapura.

\section{References}

1. Nawaratna SKS, Weilgama DJ, Wijekoon CJ, Dissanayaka M, Rajapaksha K. Cutaneous leishmaniasis, Sri Lanka. Emerging Infectious Disease 2007; 13: 1068-70. 
2. Rajapaksa US, Ihalamulla RL, Udagedera C, Karunaweera ND. Cutaneous leishmaniasis in southern Sri Lanka. Transactions of the Royal Society of Tropical Medicine and Hygiene 2007; 101: 799-803.

3. Ranawaka RR, Weerakoon HS, Opathella N, Subasinghe C. Leishmaniasis in North Central Province. Sri Lanka - three years' experiences. The Sri Lanka Journal of Dermatology 2010; 14: 4-8.

4. Siriwardana HVYD, Noyes HA, Beeching NJ, Chance ML, Karunaweera ND, Bates PA. Leishmania donovani and cutaneous leishmaniasis, Sri Lanka. Emerging Infectious Disease 2007; 13: 476-8.

5. Karunaweera ND, Pratlong F, Siriwardane HVYD, Ihalamulla RL, Dejet JP. Cutaneous leishmaniasis is caused by Leishmania donovani zymodeme MON-37. Transactions of the Royal Society of Tropical Medicine and Hygiene 2003; 97: 380-1.

6. ul Bari A, ber Rahman S. Correlation of clinical, histopathological, and microbiological findings in 60 cases of cutaneous leishmaniasis. Indian Journal of Dermatology, Venereology and Leprology 2006; 72: 28-32.

7. Al-Hucheimi SN, Sultan BA, Al-Dhalimi MA. A comparative study of the diagnosis of Old World cutaneous leishmaniasis in Iraq by polymerase chain reaction and microbiologic and histopathologic methods. International Journal of Dermatology 2009; 48: 404-8.

8. Herath $\mathrm{CH}$, Ratnatunga NV, Waduge R, Ratnayake P,
Ratnatunga CN, Ramadasa S. A histopathological study of cutaneous leishmaniasis in Sri Lanka. Ceylon Medical Journal 2010; 55: 106-11.

9. Ranawaka RR, Weerakoon HS. Randomised, double blind, comparative clinical trial on efficacy and safety of intralesional sodium stibogluconate and intralesional 7\% hypertonic sodium chloride against cutaneous leishmaniasis caused by L. donovani. Journal of Dermatologic Treatment 2010; 21: 286-93.

10. Ranawaka RR, Weerakoon HS, Opathella N. Liquid nitrogen cryotherapy on Leishmania donovani cutaneous leishmaniasis. Journal of Dermatologic Treatment 2011; 22: 241-5.

11. Marques MJ, Volpini AC, Machado-Coelho GL, MachadoPinto J, da Costa CA, Mayrink W, Genaro O, Romanha AJ. Comparison of polymerase chain reaction with other laboratory methods for the diagnosis of American cutaneous leishmaniasis: diagnosis of cutaneous leishmaniasis by polymerase chain reaction. Diagnosing Microbiology and Infectious Disease 2006; 54: 37-43.

12. Singh S. New developments in diagnosis of leishmaniasis. Indian Journal of Medical Research 2006; 123: 311-30

13. Tordini G, Giaccherini R, Pacenti L, Miracco C, Zazzi M, Zanelli G. Cutaneous leishmaniasis: usefulness of PCR on paraffin-embedded skin biopsies as part of routine investigation. Annals of Tropical Medicine and Parasitology 2007; 101: 745-9.

\title{
Geographical variation in admissions due to poisoning in Sri Lanka: a time series analysis
}

\author{
R Hanwella', S M Senanayake ${ }^{1}$, V A de Silva ${ }^{1}$ \\ (Index words: deliberate self harm, suicide, pesticides, Sri Lanka, poisoning)
}

\begin{abstract}
Objectives To carry out time series analyses of hospital admissions for poisoning between 1995-2008 in all districts in Sri Lanka to identify trends and geographical variations in the substances used in poisoning.

Methods Data of hospital admissions from 1995-2008 due to poisoning were obtained from the Annual Health Bulletins published by the Ministry of Health. Data were converted to annual rates per 100,000 population. Time trends in the rates of suicide and self-poisoning were calculated using univariate time series analysis.
\end{abstract}

Results All districts except Kilinochchi and Mullaitivu showed an increase in the rates of admissions due to poisoning with drugs, medicaments and biological substances. Colombo, Hambantota, Kalutara and Anuradhapura showed an exponential increase. Hambantota, Monaragala, Nuwara Eliya and Colombo show an increase in the rate of admissions after pesticide poisoning. All other districts showed a linear decrease. Admissions due to all types of poisoning showed a negative trend in Anuradhapura, Polonnaruwa, Ampara, Matale and Batticoloa districts. Other districts show a positive trend in the rate of admissions for all types of poisoning.

${ }^{1}$ Department of Psychological Medicine, Faculty of Medicine, University of Colombo, Sri Lanka.

Correspondence: VdeS, e-mail: <varunidesilva2@yahoo.co.uk>. Received 30 April and revised version accepted 22 August 2012. Competing interests: none declared. 